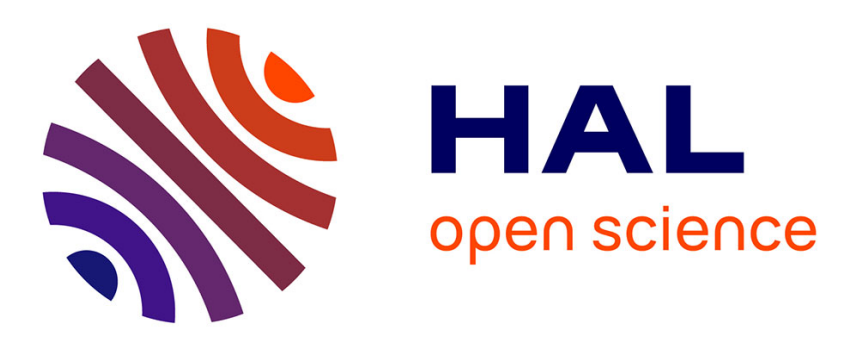

\title{
Pattern formation in the three-dimensional deformations of fibered sheets
}

Ivan Giorgio, Roman Grygoruk, Francesco Dell'isola, David J. Steigmann

\section{To cite this version:}

Ivan Giorgio, Roman Grygoruk, Francesco Dell'isola, David J. Steigmann. Pattern formation in the three-dimensional deformations of fibered sheets. Mechanics Research Communications, 2015, 69, pp.164-171. 10.1016/j.mechrescom.2015.08.005 . hal-01194318

\section{HAL Id: hal-01194318 \\ https://hal.science/hal-01194318}

Submitted on 7 Sep 2015

HAL is a multi-disciplinary open access archive for the deposit and dissemination of scientific research documents, whether they are published or not. The documents may come from teaching and research institutions in France or abroad, or from public or private research centers.
L'archive ouverte pluridisciplinaire HAL, est destinée au dépôt et à la diffusion de documents scientifiques de niveau recherche, publiés ou non, émanant des établissements d'enseignement et de recherche français ou étrangers, des laboratoires publics ou privés. 


\title{
Pattern formation in the three-dimensional deformations of fibered sheets
}

\author{
Ivan Giorgio $^{\mathrm{a}, \mathrm{d}, *}$, Roman Grygoruk ${ }^{\mathrm{b}}$, Francesco dell'Isola ${ }^{\mathrm{a}, \mathrm{d}}$, David J. Steigmann ${ }^{\mathrm{c}, \mathrm{d}}$ \\ ${ }^{a}$ Dipartimento di Ingegneria Strutturale e Geotecnica, Università di Roma "La Sapienza", 00184 Roma, Italy \\ ${ }^{b}$ Institute of Mechanics and Printing, Warsaw University of Technology 85 Narbutta, Warsaw 02-524, Poland \\ ${ }^{c}$ Department of Mechanical Engineering University of California Berkeley, CA. 94720 USA \\ ${ }^{d}$ International Research Center for the Mathematics and Mechanics of Complex Systems - MeMoCS, Università dellAquila, Italy
}

\begin{abstract}
We simulate pattern formation in the deformations of a pantographic lattice using a model of elastic surfaces that accounts for the geodesic bending of the constituent fibers. The theory predicts an unusual arrangement of coexistent phases observed in an actual lattice, manufactured by a 3D printing process, in which the fibers undergo part-wise uniform shears separated by internal transition layers controlled by geodesic bending stiffness.
\end{abstract}

Keywords: Woven fabrics, Elastic surface theory, Strain gradients

\section{Introduction}

In this work we apply a recently developed two-dimensional continuum theory of elastic surfaces (Steigmann and dell'Isola 2015) to model the main features of the deformations observed in pantographic lattices. The lattice is composed of intersecting fibers, or rods, that form two orthogonal families in an unstressed reference plane. Each member of a given fiber family regarded as a collection of parallel material curves - intersects every member of the second family at internal pivots. The resistance of these pivots to rotation about an axis aligned with the surface normal is modelled as elastic resistance to a change of angle, or shear, of the intersecting fiber families. The model also accommodates fiber stretch and three-dimensional deformations of the lattice surface. In deformations that involve a change of surface shape, the convected fibers of the pantograph flex to conform to the evolving embedding geometry. This is modelled by assigning strain energy to the normal curvatures and twists of the fibers. These variables, in turn, are determined by the second fundamental form (the curvature) of the surface, and so in this respect the present model is similar to conventional plate theory. However, in contrast to the conventional framework, the present model also accounts for the geodesic bending of the fibers. This mode of deformation occurs when, for example, the lattice is bent while deforming in a fixed plane. In the continuum theory, the effect is associated with geodesic curvature of the fibers, which in turn is controlled by the metric geometry of the surface. In particular, it involves the metric and its spatial derivatives via the Levi-Civita connection coefficients. Geodesic bending is therefore a strain-gradient effect. The pantographic substructure thus furnishes an explicit realization of straingradient elasticity (Toupin, 1964; Mindlin and Tiersten, 1962; Koiter, 1964; Germain, 1973; dell'Isola, Seppecher and Madeo, 2012; dell'Isola, Seppecher and Madeo, 2012; Ferretti, Madeo, dell'Isola and Boisse, 2013).

In Section 2 we discuss deformations observed in the so-called bias test of an actual pantographic sheet manufactured by 3D printing. These reveal a remarkable pattern of distinct phases in which the fiber shear is nearly uniform, separated by narrow internal transition layers exhibiting pronounced geodesic bending. Section 3 is devoted to a synopsis of the continuum theory developed more fully in (dell'Isola and Steigmann, 2015; Steigmann and dell'Isola, 2015). This framework may be viewed as a further development of a line of research on the continuum mechanics of fibrous materials initiated by Rivlin (1955), Green and Adkins (1970) and Pipkin $(1980,1981)$, concerning surfaces with perfectly flexible embedded fibers; Wang and Pipkin (1986), for inextensible fibers with flexural resistance; and more recent developments in the setting of generalized bulk continua (Spencer and Soldatos, 2007; Steigmann, 2012, 2015; Cheviakov et al, 2015; Nikopour and Selvadurai, 2013), biological materials (Federico et al, 2005; Grillo et al, 2012) and anisotropic mixtures (Placidi and Hutter $2006 \mathrm{a}, \mathrm{b})$. In Section 4 we discuss the details of a finiteelement solution procedure and illustrate the model with a simulation of the phase coexistence observed in the bias test, regarded as an in-plane deformation. Finally, in Section 5 we exhibit a simulation of a three-dimensional deformation that induces a similar internal strain pattern combined with substantial fiber twist.

\footnotetext{
*Corresponding author, Tel: (+39) 0644585276, Fax: (+39) 0644585292

Email address: ivan.giorgio@uniroma1.it (Ivan Giorgio)
} 
A difficult unresolved problem, not addressed here, concerns the modelling of the complex internal structure of the lattice and the implementation of a suitable homogenization procedure leading to an appropriate continuum theory (Alibert, Seppecher and dell'Isola, 2003;
Seppecher, Alibert and dell'Isola, 2011). However, to the extent that the present theory faithfully predicts the details of the observed phenomenology, it may be viewed as furnishing an appropriate target model for deeper investigations of this kind.

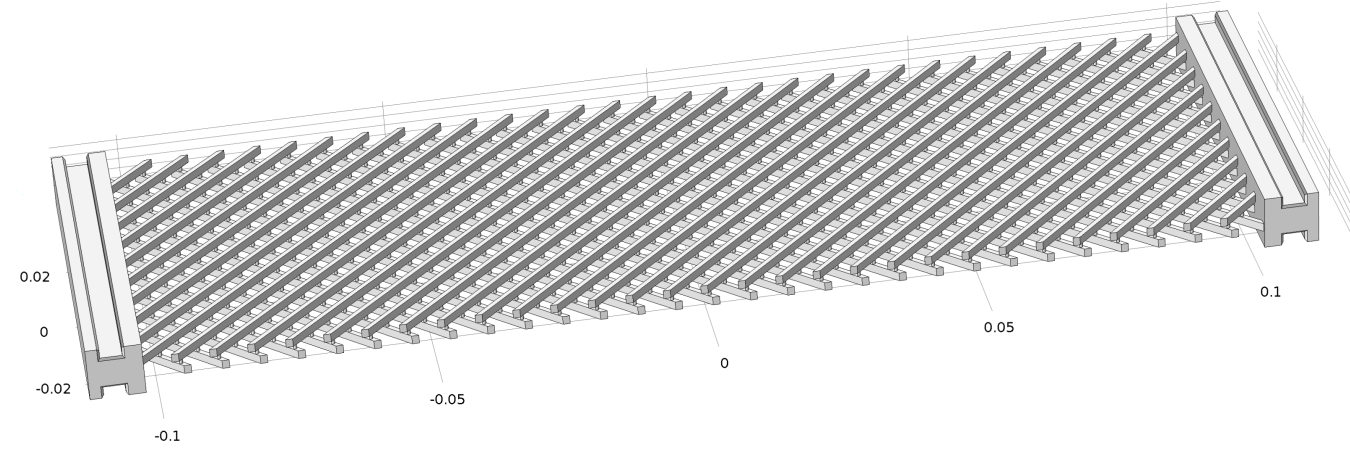

Figure 1: Pantographic lattice topology.

\section{Observations on pattern formation and phase segregation in the bias test of a uniform pan- tographic lattice}

Figure 1 depicts a pantographic lattice topology generated by SolidWorks. The same software controls the 3D printer FORMIGA P100 (EOS GmbH), which was used to construct specimens for extensional bias testing. The material used was PA 2200 (polyamide powder). The geometry of the specimen was specified by means of the STL format files (i.e., a standard file format broadly used for rapid prototyping, 3D printing and computeraided manufacturing), which provided the input to the $3 \mathrm{D}$ printer. In Figure 2 the periodic cell of the printed specimen is shown in detail.

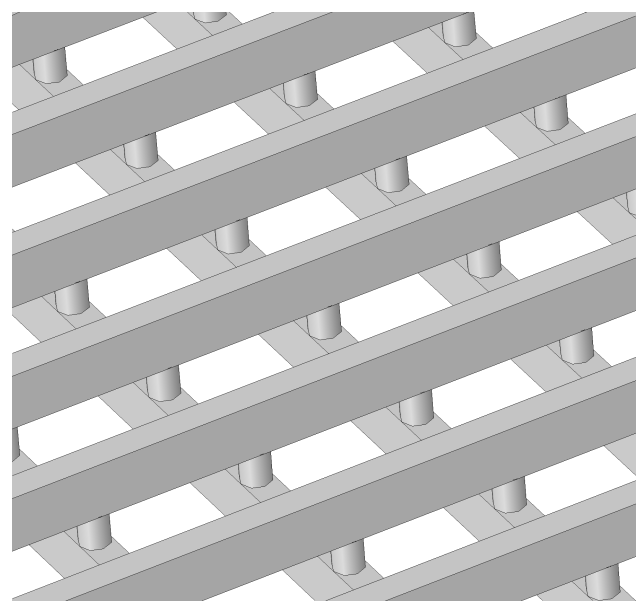

Figure 2: Details of the periodic cell of the printed specimen.
We remark that the process of 3D printing allows for the contextual construction of the specimen to be tested and the clamping devices used for connecting it to the testing apparatus.

The terminal pivots in the structure connect the terminal ends of the fibers meeting at the external boundary of the lattice. The pivots are realized by means of short connecting cylinders whose dimensions can be varied to endow the lattice with a suitably tuned shear elastic energy.

On the basis of a simplified 2D continuum model for a lattice with inextensible fibers (dell'Isola et al, 2015), it is anticipated that in the extensional bias test two triangular regions bounded by fibers remain rigid and that distinct phases exhibiting piecewise constant shear deformations emerge. This is precisely what is observed (see Fig. 3, 4).

It is further observed that the shear deformation is segregated into distinct coexistent phases separated by internal transition layers in which geodesic bending predominates. Also, some of the fibers exhibit substantial stretching, the modeling of which calls for an extended theory of the kind adopted here.

In Figure 3 the physical mesh is relatively coarse and the ratio between the rigidity of the pivots and the bending stiffness of the fibers is such that the thickness of the transition layers is limited to approximately three times the mesh size.

In Figure 4 the physical mesh is finer and the imposed elongation is larger. The shear deformation in the central region is more pronounced and the internal transition layers are thicker. 


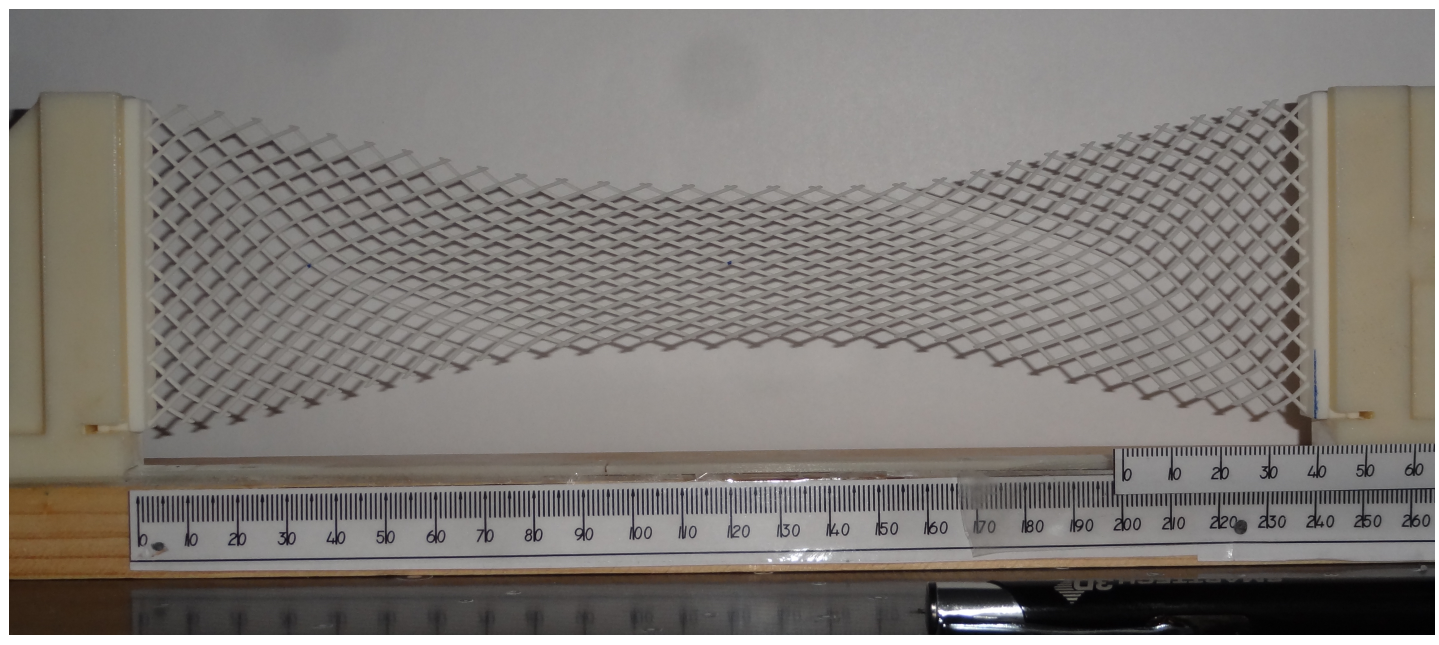

Figure 3: An example of bias extension test for pantographic lattice with coarse mesh.

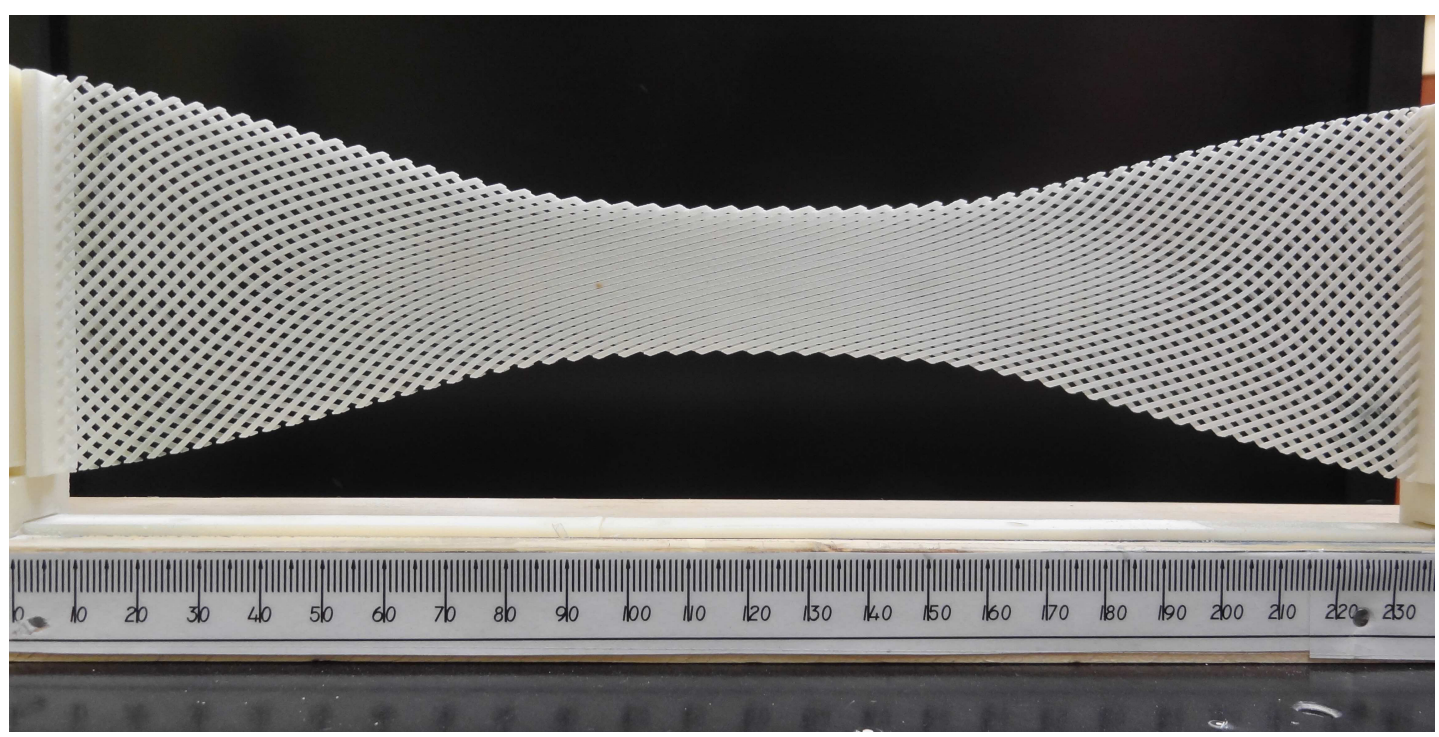

Figure 4: An example of bias extension test for pantographic lattice with finer mesh.

\section{A two-dimensional surface model incorporat- ing second-gradient elasticity}

To simulate these observations we adopt the model developed in (dell'Isola and Steigmann, 2015; Steigmann and dell'Isola, 2015), in which the pantographic sheet is regarded as an elastic surface endowed with suitable kinematic descriptors and an associated constitutive response in the form of an areal strain-energy density. The latter is assumed to depend on the stretches of the fibers and on their curvatures and twists. Further, as tangential stretch gradients appear in the constitutive equations for one-dimensional models of thin fibers that account for finite-thickness effects (Coleman, 1983; Coleman and Newman, 1988), we also include a constitutive sensitivity to the tangential derivatives of the stretches. In addition, we incorporate a non-standard constitutive sensitivity to the cross derivatives of the fiber stretches; these record the variation in the stretch of a fiber family as the (initially) orthogonal family is traversed. This effect is discussed more fully below. To make the paper reasonably self contained, we devote the remainder of the present section to a brief summary of the theory proposed in (Steigmann and dell'Isola, 2015).

The deformation of the surface is described by a map $\mathbf{r}(\mathbf{x}): \Omega \mapsto E^{3}$, where $\Omega \subset E^{2}$ is a connected plane region in the $2 \mathrm{D}$ Euclidean space $E^{2}$ and $E^{3}$ is Euclidean 3 space. Let $\mathbf{L}, \mathbf{M} \in E^{2}$ be orthogonal unit vectors defining the fiber directions prior to deformation; in this work we assume these to be uniformly distributed on $\Omega$ and hence the fibers to be initially straight. The fiber stretches $\{\lambda, \mu\}$ and fiber directions $\{\mathbf{l}, \mathbf{m}\}$ induced by the defor- 
mation are

$$
\lambda \mathbf{l}=(\nabla \mathbf{r}) \mathbf{L} \quad \text { and } \quad \mu \mathbf{m}=(\nabla \mathbf{r}) \mathbf{M}
$$

where $\nabla(\cdot)$ is the (two-dimensional) gradient with respect to $\mathbf{x}$. Here $\mathbf{l}$ and $\mathbf{m}$ are unit vectors spanning the deformed tangent plane at the material point $\mathbf{x} \in \Omega$. We use them to define the fiber shear angle $\gamma$ by

$$
\sin \gamma=\mathbf{l} \cdot \mathbf{m}
$$

Because $\{\mathbf{L}, \mathbf{M}\}$ is an orthonormal basis for $\Omega$, we may use (1) to conclude that

$$
\nabla \mathbf{r}=\lambda \mathbf{l} \otimes \mathbf{L}+\mu \mathbf{m} \otimes \mathbf{M}
$$

and thus represent the Cauchy-Green deformation tensor, $\mathbf{C}=\mathbf{F}^{t} \mathbf{F}$, in the form

$$
\mathbf{C}=\lambda^{2} \mathbf{L} \otimes \mathbf{L}+\mu^{2} \mathbf{M} \otimes \mathbf{M}+\lambda \mu \sin \gamma(\mathbf{L} \otimes \mathbf{M}+\mathbf{M} \otimes \mathbf{L}) .
$$

Further, eqs. (1) provide

$$
J \mathbf{n}=(\nabla \mathbf{r}) \mathbf{L} \times(\nabla \mathbf{r}) \mathbf{M}
$$

where $\mathbf{n}$ is the unit normal to the deformed surface, and

$$
J=\lambda \mu|\cos \gamma|
$$

is the local areal dilation induced by the deformation. Accordingly,

$$
\mathbf{l} \times \mathbf{m}=|\cos \gamma| \mathbf{n} .
$$

To describe the fiber curvatures and twist we require the second gradient $\nabla \nabla \mathbf{r}$ of the deformation. This may be represented in the form (Steigmann and dell'Isola, 2015)

$$
\begin{aligned}
& \nabla \nabla \mathbf{r}=\left(\mathbf{g}_{l}+\right.\left.K_{L} \mathbf{n}\right) \otimes \mathbf{L} \otimes \mathbf{L}+ \\
&\left(\mathbf{g}_{m}+K_{M} \mathbf{n}\right) \otimes \mathbf{M} \otimes \mathbf{M}+ \\
&(\mathbf{\Gamma}+T \mathbf{n}) \otimes(\mathbf{L} \otimes \mathbf{M}+\mathbf{M} \otimes \mathbf{L})
\end{aligned}
$$

with

$$
\mathbf{g}_{l}=\lambda^{2} \eta_{l} \mathbf{p}+(\mathbf{L} \cdot \nabla \lambda) \mathbf{l}, \quad \mathbf{g}_{m}=\mu^{2} \eta_{m} \mathbf{q}+(\mathbf{M} \cdot \nabla \mu) \mathbf{m}
$$

and

$$
\boldsymbol{\Gamma}=(\mathbf{L} \cdot \nabla \mu) \mathbf{m}+\lambda \mu \phi_{m} \mathbf{q}=(\mathbf{M} \cdot \nabla \lambda) \mathbf{l}+\lambda \mu \phi_{l} \mathbf{p},
$$

in which $\eta_{l}$ and $\eta_{m}$ are the geodesic curvatures of the deformed fibers, $\phi_{l}$ and $\phi_{m}$ are the so-called Tchebychev curvatures, and

$$
\mathbf{p}=\mathbf{n} \times \mathbf{l}, \quad \mathbf{q}=\mathbf{n} \times \mathbf{m}
$$

define the orthogonal directions of the fibers on the deformed surface. The second of eqs. (10) expresses the equality of the cross derivatives of the deformation in the two fiber directions and therefore furnishes a compatibility condition. Further,

$$
K_{L}=\lambda^{2} \kappa_{l}, \quad K_{M}=\mu^{2} \kappa_{m} \quad \text { and } \quad T=\lambda \mu \tau,
$$

where $\kappa_{l}$ and $\kappa_{m}$ are the normal curvatures of the deformed fibers and $\tau$ measures the twist of the deformed surface. These are non-zero, collectively or individually, if and only if the deformation is such as to engender curvature of the surface in 3-space. Accordingly, they describe those parts of the fiber curvatures that can be attributed to surface flexure, whereas the geodesic curvatures represent the components of fiber curvatures in the tangent planes of the deformed surface. The latter arise from the surface strain and the strain gradient; the explicit expressions are (Steigmann and dell'Isola, 2015)

$$
J \eta_{l}=\mathbf{L} \cdot \nabla(\mu \sin \gamma)-\mathbf{M} \cdot \nabla \lambda
$$

and

$$
J \eta_{m}=\mathbf{L} \cdot \nabla \mu-\mathbf{M} \cdot \nabla(\lambda \sin \gamma)
$$

whereas the Tchebychev curvatures are given by (Steigmann and dell'Isola, 2015)

$$
J \phi_{l}=J \eta_{m}+\lambda \mathbf{M} \cdot \nabla(\sin \gamma)
$$

and

$$
J \phi_{m}=J \eta_{l}-\mu \mathbf{L} \cdot \nabla(\sin \gamma) .
$$

The latter formulas also involve the strain and strain gradient. When combined with either of eqs. (10), they yield the conclusion that $\boldsymbol{\Gamma}$ ultimately depends only on the fiber stretches and their cross derivatives $\mathbf{L} \cdot \nabla \mu$ and $\mathbf{M} \cdot \nabla \lambda$; and, moreover, that $\boldsymbol{\Gamma}$ vanishes if and only if the cross derivatives vanish together whenever $J>0$. These statements are proved in (dell'Isola and Steigmann, 2015). In the alternative case $(J=0)$ the fibers collapse locally onto a curve. This degenerate case is penalized by our constitutive assumptions.

All constitutive information in the model is codified in an areal strain-energy density $W$ that depends on the first and second gradients of the deformation. To nondimensionalize the variables appearing in this function it is necessary to introduce a local length scale. Candidates for this are the thickness of the actual sheet represented by our surface model, the characteristic spacing of the internal pivot points of the actual pantographic lattice, or the widths of the constituent fibers. If any of these is used as the length scale, then in typical applications the nondimensionalized vectors $\mathbf{g}_{l}, \mathbf{g}_{m}$ and $\boldsymbol{\Gamma}$ are so small that the dependence of the strain energy on them is quadratic at leading order, assuming the associated couple stresses and bending/twisting moments to vanish when the fibers are straight and untwisted. A simple strain-energy function of this type, incorporating the orthotropic symmetry conferred by the initial fiber geometry, is

$$
\begin{aligned}
& W=w(\lambda, \mu, J)+\frac{1}{2}\left(A_{l}\left|\mathbf{g}_{l}\right|^{2}+A_{m}\left|\mathbf{g}_{m}\right|^{2}+\right. \\
& \left.A_{\Gamma}|\boldsymbol{\Gamma}|^{2}+k_{L} K_{L}^{2}+k_{M} K_{M}^{2}+k_{T} T^{2}\right),
\end{aligned}
$$


where the coefficients $A_{l, m, \Gamma}$ and $k_{L, M, T}$ are constants; this form is assumed for the sake of definiteness and tractability. Other forms are, of course, possible. In particular, we might separate out the effects of geodesic curvature and tangential stretch gradient in $\mathbf{g}_{l}$ or $\mathbf{g}_{m}$, and assign different elastic moduli to each. Here we take $A_{l, m, \Gamma}$ and $k_{L, M, T}$ to be strictly positive and observe that the part of the energy depending on the second gradient $\nabla \nabla \mathbf{r}$ is then non-negative, vanishing if and only if $\mathbf{g}_{l}$, $\mathbf{g}_{m}, \boldsymbol{\Gamma}, K_{L}, K_{M}$ and $T$ all vanish simultaneously. In view of (8) it is thus a convex function of $\nabla \nabla \mathbf{r}$, which is enough to secure the existence of energy-minimizing deformations in conservative boundary-value problems via the direct method of the calculus of variations (Ball, Currie and Olver, 1981). Further, the existence of a solution to the weak form of the equilibrium equations for similar problems in second-gradient elasticity has been proved in (Healey and Krömer, 2008), albeit under hypotheses slightly more stringent than those satisfied by the present model.

The constitutive sensitivities to geodesic and normal curvatures are readily understood in terms of the mechanics of the lattice substructure. Specifically, fibers are expected to offer resistance to any mode of bending, be it of the geodesic type or the type induced by surface flexure. Regarding fiber twist, in the present model this is determined by the twist of the surface because of the presumed connectivity of the lattice; that is, both families of intersecting fibers are assumed to pivot about the surface normal, which of course evolves in the course of a general three-dimensional deformation. This constraint implies that fiber twist is controlled entirely by surface geometry and is therefore not an independent kinematic variable (Steigmann and dell'Isola, 2015).

We have already discussed grounds for introducing elastic resistance to the tangential derivatives of the fiber stretches. The influence of the cross derivatives, represented by the vector $\boldsymbol{\Gamma}$, may be motivated by considering a fiber with stretch equal to unity at a particular point. While this particular fiber does not contribute to the energy density, a non-zero cross derivative imposes extensional strain on the neighboring parallel fibers and therefore generates an energy in the lattice in a neighborhood of the considered point. The average energy in the neighborhood is then assigned by the local strain-energy function to the point in question. Further, we find that inclusion of this effect is necessary to secure the convexity of the strain-energy function with respect to $\nabla \nabla \mathbf{r}$; its absence, corresponding to $A_{\Gamma}=0$, yields a strainenergy function that is only positive semi-definite in the second gradient of deformation and hence ill-posed from the standpoint of energy minimization.

For the strain-dependent function $w$, we adopt

$$
w(\lambda, \mu, J)=\frac{1}{2}\left(E_{L} \epsilon_{L}^{2}+E_{M} \epsilon_{M}^{2}\right)-G(\ln J+1-J),
$$

where

$$
\epsilon_{L}=\frac{1}{2}\left(\lambda^{2}-1\right), \quad \epsilon_{M}=\frac{1}{2}\left(\mu^{2}-1\right)
$$

are the extensional fiber strains and $E_{L, M}$ and $G$ are positive constants. This energy does not include a term proportional to $\epsilon_{L} \epsilon_{M}$ and therefore does not accommodate a Poisson effect with respect to the fiber axes. Poisson effects are generally non-negligible in woven fabrics due to fiber crimping and de-crimping (dell'Isola and Steigmann, 2015; Steigmann and dell'Isola, 2015; Atai and Steigmann, 2014), a mechanism that is absent in pantographic lattices. Finally, the term involving $J$ penalizes fiber collapse $(J \rightarrow 0)$ by imposing unbounded growth of the attendant energy, whereas the remaining terms are appropriate for small-to-moderate fiber strains.

In the case of large fiber strains, it is possible to include the effects of damage and plasticity, as described, for example, in (Placidi, 2014; Rinaldi, 2015; Yang et al, 2011) and (Placidi, in press), respectively. For other types of fibers configurations the symmetry analysis performed in (Eremeyev and Pietraszkiewicz, 2006) may be useful for construction of the 2D strain energy density.

Our solution procedure, discussed in Section 4, is a finite-element scheme based on the weak form of the equilibrium equations derived from the proposed strainenergy function. For the sake of brevity we do not exhibit this system here but instead refer the interested reader to (Steigmann and dell'Isola, 2015), where both the weak and strong formulations may be found. Recent applications of the finite element method and related methods to second-gradient elasticity are discussed in (Fischer et al, 2010 and 2011; Rudrarajua et al, 2014; Cazzani et al, 2014; Greco and Cuomo, 2013; Turco and Aristodemo, 1998).

\section{Finite-element solution}

The plane region $\Omega$ considered here is a rectangle whose sides are in the ratio 1:3.

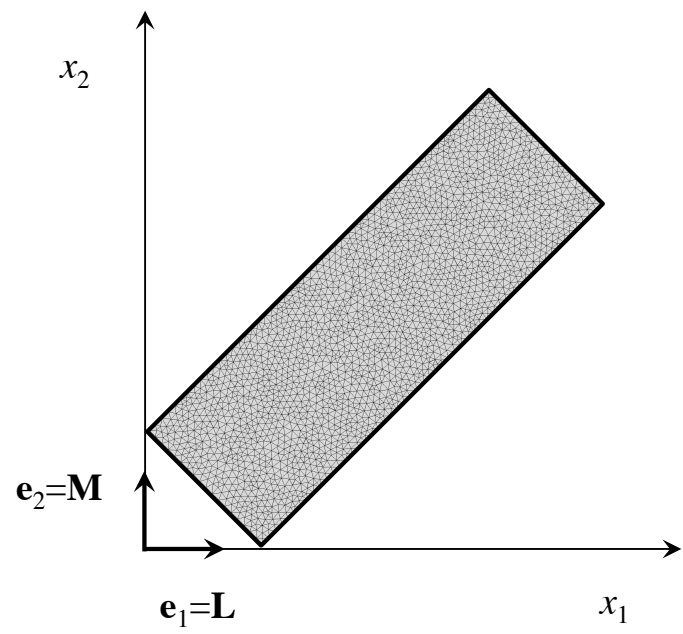

Figure 5: The simulated fibered sheet and a plot of the finite element mesh used. 
The two orthogonal families of fibers are arranged so as to form an angle of 45 degrees with the sides of this region; thus, placing the rectangle according to the bisector of the first quadrant of the reference plane, the unit vectors $\mathbf{L}, \mathbf{M}$ are simply the basis elements $\mathbf{e}_{1}, \mathbf{e}_{2}$ associated with a Cartesian system (see Fig. 5). In the numerical simulations, a non-dimensional form of the problem is achieved by normalizing the strain energy with respect to a reference stiffness; and the lengths with respect to the length of the shorter edge. Using a tilde to denote the non-dimensional quantities, the normalized moduli employed in the numerical simulations are:

- $\tilde{E}_{L}=\tilde{E}_{M}=100$;

- $\tilde{G}=0.02$;

- $\tilde{A}_{l}=\tilde{A}_{m}=0.001$

- $\tilde{A}_{\Gamma}=0.01$

- $\tilde{k}_{L}=\tilde{k}_{M}=0.001$

- $\tilde{k}_{T}=0.01$

We imposed displacement boundary conditions on the opposing short sides of the lattice. In particular, the displacement $\mathbf{u}=\mathbf{r}(\mathbf{x})-\mathbf{x}$ is zero on one edge, while on the other we impose the rigid displacement $\mathbf{u}=u_{i} \mathbf{e}_{i}$, with

$$
\left\{\begin{array}{l}
u_{1}=u_{0}+(s-1 / 2)(\cos (\theta)-1) \cos (\pi / 4) \\
u_{2}=u_{0}-(s-1 / 2)(\cos (\theta)-1) \sin (\pi / 4) \\
u_{3}=(s-1 / 2) \sin (\theta)
\end{array}\right.
$$

where $s$, which varies from 0 to 1 , is a parameter, $\theta$ is a rotation angle with respect to the longitudinal axis of rectangle, and $u_{0}$ is a stretching displacement along the same axis.

Because the normal derivative of position is not assigned, then implicitly we are assigning (in the weak sense) zero double force on edges where position is assigned. Elsewhere we assign zero double force and zero traction.

We note that, in the finite element analysis performed with the commercial software COMSOL Multiphysics, we employed Argyris elements having $C^{1}$ continuity; these can be used to properly approximate functions in the Sobolev space $H^{2}$, which is the appropriate space for problems of the present kind involving the second gradient of the placement. Indeed, in Argyris triangles, the interpolation functions are quintic polynomials and the 21 degrees of freedom characterizing this element are chosen to be the values of the considered field (or each component of a vector field), the first and second derivatives at the vertices, and the normal derivative at the middle of the sides.

In a first simulation we assigned $u_{0}=0.5$ and $\theta=0$. The predicted deformation, depicted by selected fiber trajectories in the deformed configuration in Figure 6, shows remarkable agreement with that actually observed and discussed in Section 2, particularly with respect to the distinct phases of uniform fiber shear separated by narrow internal transition layers.

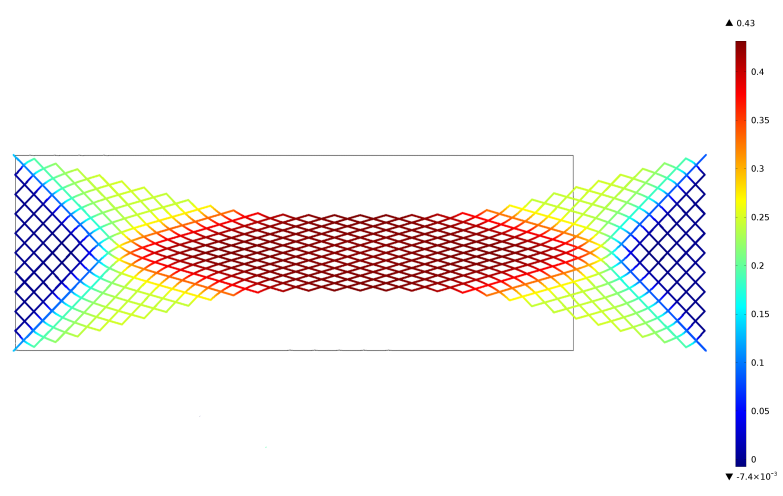

Figure 6: Numerical simulation of the bias extension test. The colors indicate the shear strain relative to the initial fiber axes.

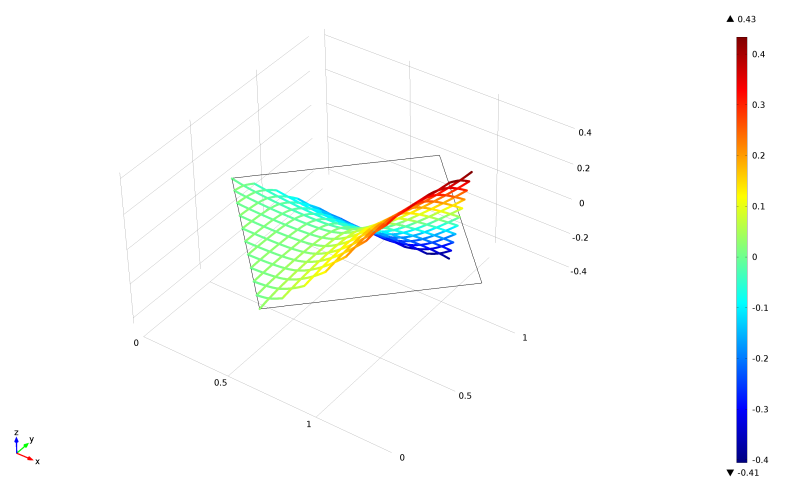

Figure 7: An example of torsion of a square sheet ( $\theta=60$ degrees). The colors indicate the out-of-plane component of displacement, $u_{3}$.

\section{A three-dimensional example}

We close with some examples in which the surface is caused to bend, stretch and twist in three dimensions by the relative rotation and translation of opposing boundaries where position is assigned (see Figures 7-12).

The effect is to produce together with elongation also a substantial fiber twist with the coexistent distribution of shear phases remaining embedded in the surface. As the novelty of the presented model consists in the dependence of deformation energy on geodesic curvature and in its coupling with twisting and elongation, we present in figg. 8-12 the calculated shear deformation (as function of Lagrange coordinates) relative to the cases:

1. where the rectangular specimens may reduce to squares or have one side two or three times longer than the shorter; 
2. where the imposed twist relative rotation (amounting to 60 degrees) of opposing boundaries is added to a stretching (see figg. 8-10);

3 . where the imposed twist relative rotation (amounting to 45 degrees) of opposing boundaries is added to a global specimens shear relative displacement (see figg. 11, 12).

As expected the effect of the relative twist rotation on fiber extension and shear, is enhanced when the ratio of the two sides of the rectangles is closer to one. Moreover it can be seen in the presented plots that the shear deformation patterns determined by geodesic bending, and in particular the transition zones separating different shear regions, are greatly influenced by the interaction of elongation, global and local shear and twist. Corroborative experimental evidence for these simulations is not currently available, but will be sought as part of our ongoing investigation into the complex deformations of pantographic lattices. In all previous examples the deformation is supported by reactive corner forces similar to those of classical plate-bending theory, except that here these forces may have also components tangential to the deformed surface, in addition to normal components, due to the non-standard geodesic bending resistance of the lattice (Steigmann and dell'Isola, 2015).
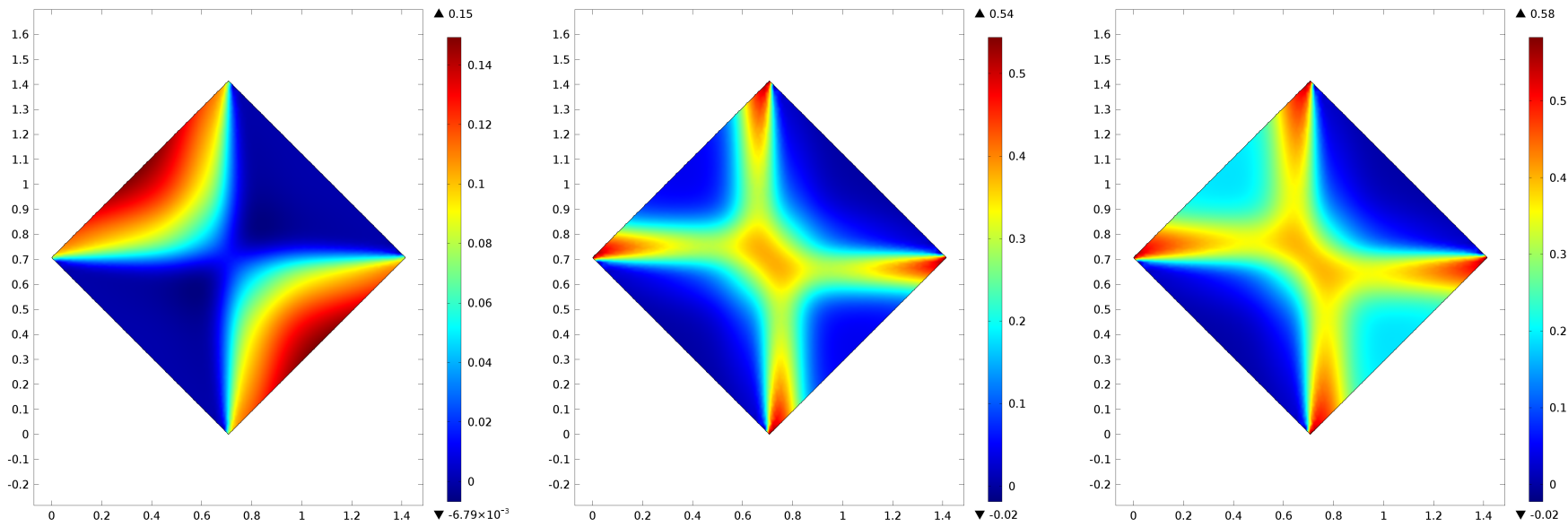

Figure 8: The shear strain relative to the initial fiber axes; from left to right: only twist, only stretching, both $\left(u_{0}=0.1, \theta=60\right.$ degrees $)$. Fibered sheet whose sides are in the ratio 1:1.
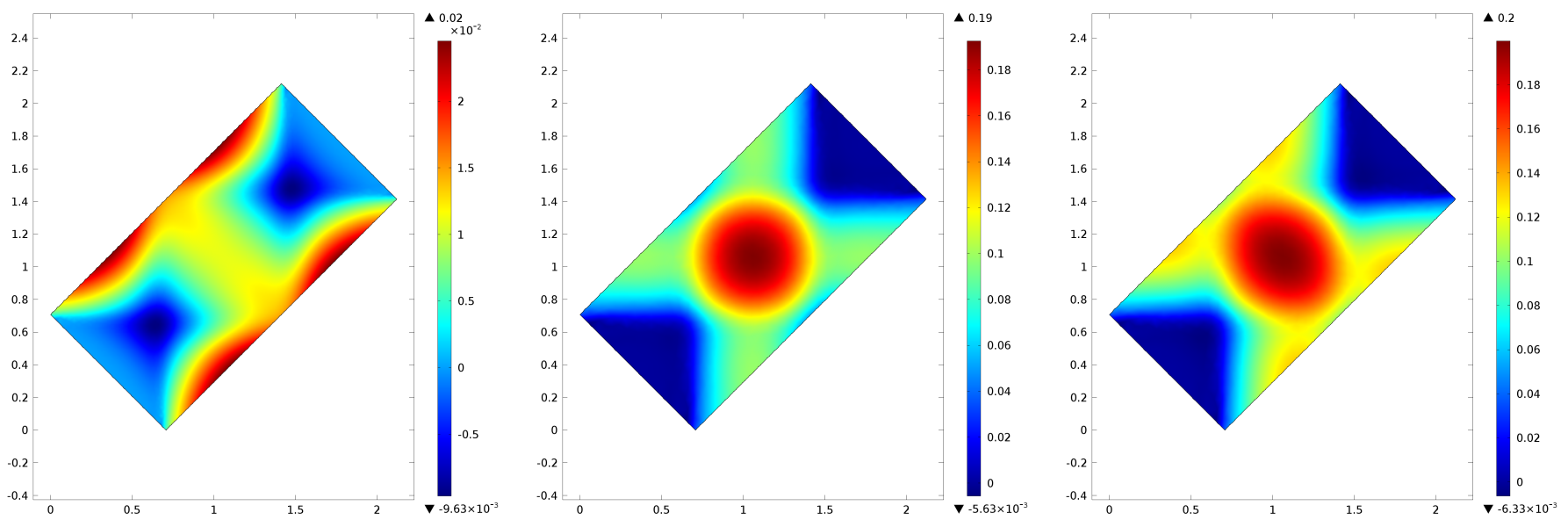

Figure 9: The shear strain relative to the initial fiber axes; from left to right: only twist, only stretching, both $\left(u_{0}=0.1, \theta=60\right.$ degrees $)$. Fibered sheet whose sides are in the ratio 1:2. 

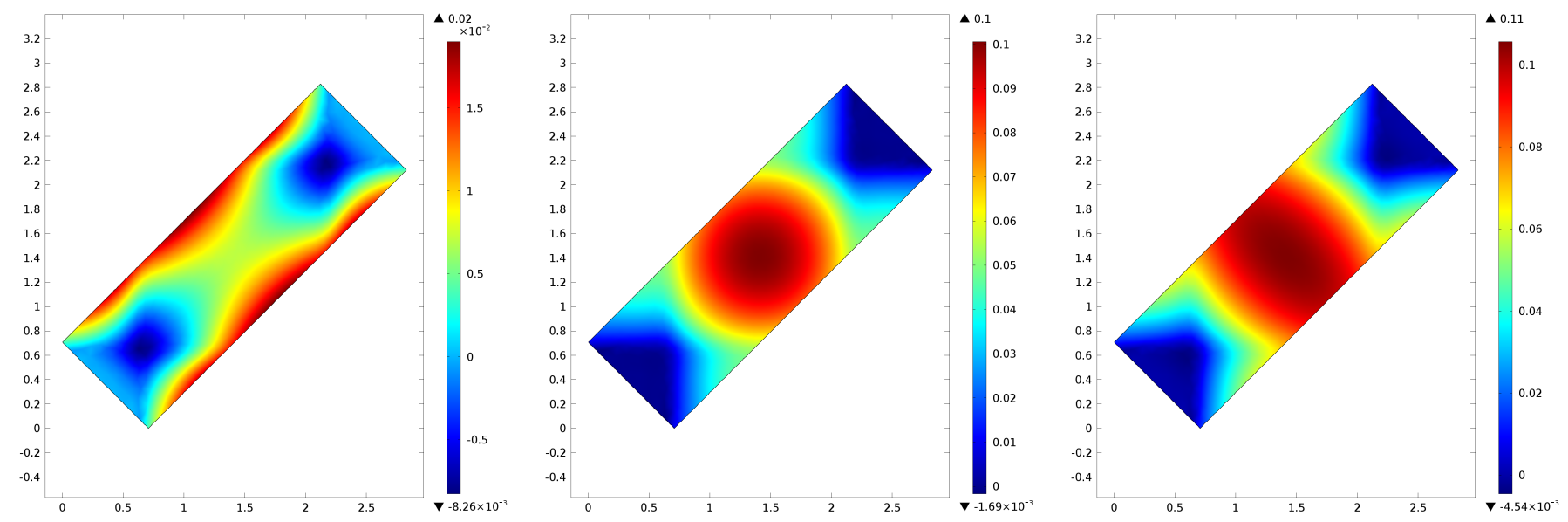

Figure 10: The shear strain relative to the initial fiber axes; from left to right: only twist, only stretching, both $\left(u_{0}=0.1, \theta=60\right.$ degrees $)$. Fibered sheet whose sides are in the ratio 1:3.
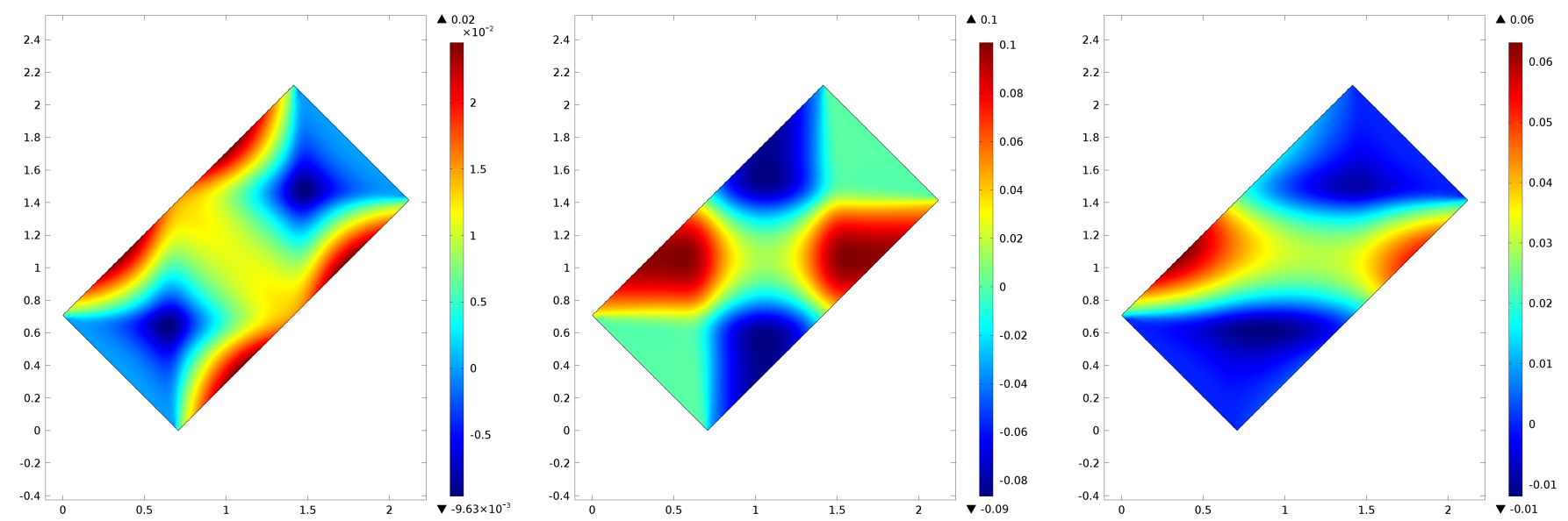

Figure 11: The shear strain relative to the initial fiber axes; from left to right: only twist, only shear, both $\left(u_{0}=0.1, \theta=45\right.$ degrees). Fibered sheet whose sides are in the ratio $1: 2$.
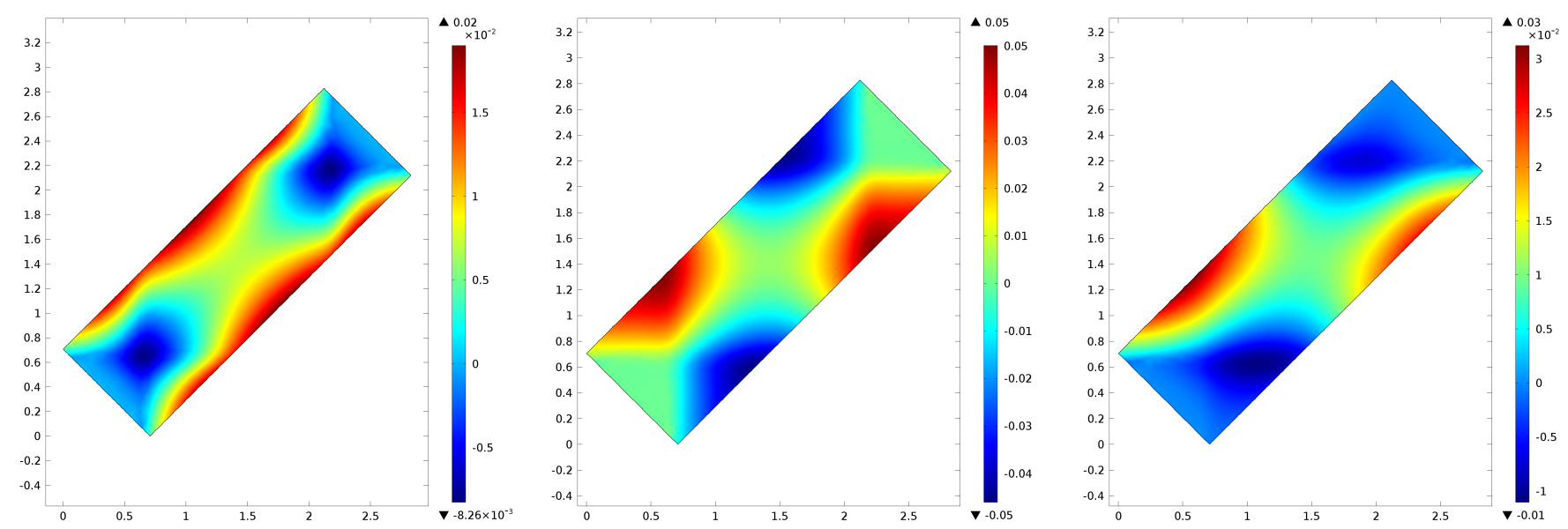

Figure 12: The shear strain relative to the initial fiber axes; from left to right: only twist, only shear, both $\left(u_{0}=0.1, \theta=45\right.$ degrees $)$. Fibered sheet whose sides are in the ratio 1:3. 


\section{Conclusions}

In this paper, we consider an elastic fibered sheet, in which the fibers are arranged in a pantographic lattice. This two-dimensional continuum theory of elastic surfaces which accounts for the geodesic bending of the constituent fibers, and recently developed by (Steigmann and dell'Isola, 2015), is employed to simulate pattern formation in the deformations of a pantographic lattice. The theoretical framework may be viewed as a further development of a line of research on the continuum mechanics of fibrous materials originated by Rivlin (1955), Green and Adkins (1970) and Pipkin $(1980,1981)$ concerning surfaces with perfectly flexible embedded fibers, and Wang and Pipkin (1986) for inextensible fibers with flexural resistance. The theory predicts an unusual arrangement of coexistent phases observed in an actual lattice, manufactured by a 3D printing process, in which the fibers undergo part-wise uniform shears separated by internal transition layers controlled by geodesic bending stiffness. The model is illustrated with a simulation of the phase coexistence observed in the bias test, regarded as an in-plane deformation. Finally, some simulations of a three-dimensional deformation that induces a similar internal strain pattern combined with substantial fiber twist are presented on rectangular samples whose sides are in different ratios.

Acknowledgments: The work of IG and Fd'I was sponsored by M\&MoCS: the Italian Center for the Mathematics and Mechanics of Complex Systems. DJS gratefully acknowledges his appointment as a Visiting Research Professor at the Università di Roma "La Sapienza" during the course of this research.

\section{References}

Alibert, J., Seppecher, P., and dell'Isola, F., 2003, Truss modular beams with deformation energy depending on higher displacement gradients. Math. Mech. Solids 8, 51-73.

Atai, A. and Steigmann, D.J., 2014, Numerical analysis of wrinkled, anisotropic, nonlinearly elastic membranes. Mech. Res. Comm. 57, 1-5.

Ball, J.M., Currie, J.C. and Olver, P.J., 1981, Null Lagrangians, weak continuity, and variational problems of arbitrary order. J. Functional Anal. 41, 135-174.

Cazzani, A. and Malagù, M. and Turco, E., 2014, Isogeometric analysis: a powerful numerical tool for the elastic analysis of historical masonry arches. Contin. Mech. Thermodyn. (DOI: 10.1007/s00161-014-0409-y).

Cheviakov, A. F. and Ganghoffer, J.-F. and St. Jean, S., 2015, Fully non-linear wave models in fiber-reinforced anisotropic incompressible hyperelastic solids. Internat. J. Non-Linear Mech. (DOI: 10.1016/j.ijnonlinmec.2015.01.006).
Coleman, B.D., 1983, Necking and Drawing in Polymeric Fibers Under Tension. Arch. Rational Mech. Anal. 83, 115-137.

Coleman, B.D. and Newman, D.C., 1988, On the Rheology of Cold Drawing: I. Elastic Materials, J. Polym. Sci. B, Polymer Physics. 26, 1801-1822.

dell'Isola, F., Seppecher, P. and Madeo, A., 2012, Beyond Euler-Cauchy Continua: The structure of contact actions in $\mathrm{N}$-th gradient generalized continua: a generalization of the Cauchy tetrahedron argument. Variational Models and Methods in Solid and Fluid Mechanics CISM Courses and Lectures vol. 535, pp. 17-106.

dell'Isola, F., Seppecher, P. and Madeo, A., 2012, How contact interactions may depend on the shape of Cauchy cuts in N-th gradient continua: approach "à la D'Alembert", Zeitschrift für Angewandte Mathematik und Physik 63, 1119-1141.

dell'Isola, F. and Steigmann, D.J., 2015, A twodimensional gradient-elasticity theory for woven fabrics. J. Elasticity 18, 113-125.

dell'Isola, F., d'Agostino, M., Madeo, A. and Steigmann, D.J., 2015, Minimization of shear energy in two-dimensional continua with two orthogonal families of inextensible fibers: the case of the standard bias extension test. J. Elasticity (doi: 10.1007/s10659-015-9536-3).

Eremeyev, V. A., and Pietraszkiewicz, W., 2006, Local symmetry group in the general theory of elastic shells. Journal of Elasticity, 85 (2), 125-152.

Federico, S. and Grillo, A. and La Rosa, G. and Giaquinta, G. and Herzog, W., 2005, A Transversely Isotropic, Transversely Homogeneous MicrostructuralStatistical Model of Articular Cartilage, J. Biomechanics, 38 (10), 2008-2018.

Ferretti, M., Madeo, A., dell'Isola, F. and Boisse, P., 2013, Modeling the onset of shear boundary layers in fibrous composite reinforcements by second-gradient theory. Zeitschrift fur Angewandte Mathematik und Physik. (http://dx.doi.org/10.1007/s00033-013-0347-8).

Fischer, P., Mergheim, J. and Steinmann, P., 2010, On the $\mathrm{C} 1$ continuous discretization of non-linear gradient elasticity: A comparison of NEM and FEM based on Bernstein-Bézier patches. Int. J. Numer. Meth. Engng. 82,1282-1307.

Fischer, P., Klassen, M., Mergheim, J., Steinmann, P. and Müller, R. 2011, Isogeometric analysis of 2D gradient elasticity. Comput Mech. 47, 325-334.

Germain, P., 1973, The method of virtual power in continuum mechanics, part 2: microstructure. SIAM J. Appl. Math. 25, 556-575.

Greco, L. and Cuomo, M., 2013, B-Spline interpolation of Kirchhoff-Love space rods. Comput. Methods Appl. Mech. Engrg. 256, 251-269.

Green, A.E. and Adkins, J.E., 1970, Large elastic deformations. Oxford University Press.

Grillo, A. and Federico, S. and Wittum, G., 2012. Growth, Mass Transfer, and Remodeling in 
Fiber-Reinforced, Multi-Constituent Materials. Internat. J. Non-Linear Mech. 47(2), 388-401, DOI: 10.1016/j.ijnonlinmec.2011.09.026

Healey, T.J. and Krömer, S., 2008, Injective weak solutions in second gradient nonlinear elasticity. ESAIM: Control, Optimisation and Calculus of Variations (DOI: 10.1051/cocv:2008050).

Koiter, W.T., 1964, Couple-stresses in the theory of elasticity. Proc. Knononklijke Nederlandse Akademie van Wetenschappen B67, 17-44.

Mindlin, R.D. and Tiersten, H.F., 1962, Effects of couple-stresses in linear elasticity. Arch. Rational Mech. Anal. 11, 415-448.

Nikopour, H. and Selvadurai, A.P.S., 2013, Torsion of a layered composite strip. Composite Structures, 95, $1-4$.

Pipkin, A.C., 1980, Some developments in the theory of inextensible networks. Q. Appl. Math. 38, 343.

Pipkin, A.C., 1981, Plane traction problems for inextensible networks. Q.J. Mech. appl. Math. 34, 415.

Placidi, L., 2014, A variational approach for a nonlinear one-dimensional damage-elasto-plastic secondgradient continuum model. Contin. Mech. Thermodyn. (DOI: 10.1007/s00161-014-0405-2).

Placidi, L., 2014, A variational approach for a nonlinear 1-dimensional second gradient continuum damage model. Contin. Mech. Thermodyn. (DOI: 10.1007/s00161-14-0338-9).

Placidi, L. and Hutter, K., 2006a, An Anisotropic Flow Law for Incompressible Polycrystalline Materials. Z. Angew. Math. Phys. 57, 160-181. (DOI: 10.1007/s00033-005-0008-7).

Placidi, L. and Hutter, K., 2006b, Thermodynamics of Polycrystalline materials treated by the Theory of Mixtures with Continuous Diversity. Contin. Mech. Thermodyn. 17, 409-451.

Rinaldi, A. and Placidi, L., 2015, A microscale second gradient approximation of the damage parameter of quasi-brittle heterogeneous lattices. Z. Angew. Math. Mech. 94, 862-877 (DOI: 10.1002/zamm.201300028).
Rivlin, R.S., 1955, Plane strain of a net formed by inextensible cords. J. Ration. Mech. Anal. 4, 951-974.

Rudrarajua, S., Van der Venb, A. and Garikipati, K., 2014, Three-dimensional isogeometric solutions to general boundary value problems of Toupin's gradient elasticity theory at finite strains. Comput. Methods Appl. Mech. Engrg. 278, 705-728.

Seppecher, P., Alibert, J. and dell'Isola, F., 2011, Linear elastic trusses leading to continua with exotic mechanical interactions. Journal of Physics: Conference Series 319

Spencer, A.J.M. and Soldatos, K.P., 2007, Finite deformations of fibre-reinforced elastic solids with fibre bending stiffness. Int. J. Non-Linear Mech. 42, 355-368.

Steigmann, D.J., 2012, Theory of elastic solids reinforced with fibers resistant to extension, flexure and twist. Int. J. Non-linear Mech. 47, 734-742.

Steigmann, D.J., 'Effects of fiber bending and twisting resistance on the mechanics of fiber-reinforced elastomers', in: CISM Course on Nonlinear Mechanics of Soft Fibrous Tissues (L. Dorfmann and R.W. Ogden, eds.). Springer, Wien and New York, pp. 269-305, 2015.

Steigmann, D.J. and dell'Isola, F., 2015, Mechanical response of fabric sheets to three-dimensional bending, twisting and stretching. Acta Mechanica Sinica (doi: 10.1007/s10409-015-0413-x).

Toupin, R.A., 1964, Theories of elasticity with couple stress. Arch. Rational Mech. Anal. 17, 85-112.

Turco, E. and Aristodemo, M., 1998, A threedimensional B-spline boundary element, Comput. Methods Appl. Mech. Engrg. 155, 119-128.

Yang, Y., Ching, W.Y. and Misra, A., 2011, Higherorder continuum theory applied to fracture simulation of nano-scale intergranular glassy film. Journal of Nanomechanics and Micromechanics, 1:6071.

Wang, W.-B. and Pipkin, A.C., 1986, Inextensible networks with bending stiffness. Q. Jl. Mech. appl. Math. 39, 343-359.

Wang, W.-B. and Pipkin, A.C., 1986, Plane deformations of nets with bending stiffness. Acta Mech. 65, 263-279. 